\title{
Review of Wide Bandwidth Low Power Low NOISE Amplifier for Modern Wireless Communication
}

\author{
Bhavna Prajapati \\ PG Scholar's NIIST Bhopal
}

\author{
Swapnil Jain \\ HoD EC \\ NIIST Bhopal
}

\author{
Braj Bihari Soni \\ Asst Prof \\ NIIST Bhopal
}

\begin{abstract}
Recently, Low noise amplifier versatile used in modern wireless communication like Wi-Max, WLAN, GSM, Bluetooth and satellite communication. Low Noise amplifier have important feature like amplify the signal with rejection of noise. Low noise amplifier in modern communication used as filter with amplifier. In recent scenario low noise amplifier available in wide band, single band, multi-band frequency of application. In present days low noise amplifier the also reduces the reflection of signal exist by elements and connecting interface inside the amplifier. Low noise amplifier available with high gain, noise rejection and with less power consumption. In this paper review the work of past decades done in low noise amplifier. Low Noise Amplifier (LNA) is versatile used as a broadband mixer, low noise amplifier, power amplifier and Darlington amplifier, active balunes, multiband amplifier. Today technology required high speed of transmission efficiency with small power consumption and less utilization of elements in proposed amplifier, Low Noise Amplifier (LNA) products full fill all requirement of modern wireless communications, so that review and discussion, future requirement of technology is needed to discuss. In this paper discusses issues of low noise amplifier, its application, issues and recent trends. In this paper review some techniques of Low Noise Amplifier (LNA) to improve perform and surveyed almost all the Possible Work of Past Decades.
\end{abstract}

\section{Keywords}

Ultra-wideband (UWB), Differential mode of application, cross coupled feedback, Direct -Coupled amplifier.

\section{INTRODUCTION}

CONTINUOUS scaling of CMOS technology keeps driving the innovation of RFICs with higher integration level and lower cost. Significant efforts on the study of both devices and circuits also substantiate the wireless communication systems operating toward higher frequencies. Using the $\mathrm{K}$ band $(18-26.5 \mathrm{GHz})$ for short-range and high data-rate wireless communication and anti-collision radars is recently of great interest to both industry and academia [1]-[6]. Similar with other portable wireless applications, low-power design is a critical Issue [6]. Design of RF LNAs consists of two major parts, namely selection of transistor geometry and bias point, and also determination of circuit topology including the matching networks. The characteristics of transistors play a critical role, since the core circuit is composed of only a few transistors in most cases. In addition, a simple circuit topology is often preferred to prevent the unpredicted parasitic effects from the complicated layout.

\section{REVIEW OF TECHNIQUES}

For improvement of the performance and linearity of of low noise amplifier many number of techniques used in past decades like Wide Range Derivative Superposition Technique, Direct-Coupled Amplifier Topology [2], Resistive shunt feedback topology and Matching T-network sections, Forward Combining Technique, Gate-Inductive Gain-Peaking Technique, Si-Ge Bi-CMOS technology, switched multi-tap transformer and inductively degenerated this all techniques discuss in details in below section.

\subsection{Wide Range Derivative Superposition Technique}

In this technique presents an L-band highly linearity differential low noise amplifier (LNA), a wide range derivative superposition technique is used to elimininate reflection and contain of noise of amplifier. By this reduction it reduces the third-order intercept point (IP3), and simultaneously, reduces the third-order inter-modulation distortion (IMD3) over a broad impedance bandwidth. For further improvement in linearity, noise performance of amplifier next technique is discussed in next section.

\subsection{Direct-Coupled Amplifier Topology}

A direct-coupled technique is used in amplifier to enhance linearity and stability, technique is shown in Fig. 1. This technique has demonstrated in [1], gives $2.5 \mathrm{~dB}$ noise figure and $3-\mathrm{dB}$ bandwidths up to $6 \mathrm{GHz}[1]$. The technique consists of two gain stages. The first stage is a common-emitter amplifier of HBT transistors, Q1.The second stage is a feedback amplifier consists of HBT Darlington connected transistors, Q2 and Q3, series feedback resistor, shunt feedback resistor, bias resistor load resistor, and output matching resistor. The first stage acts as a low noise common emitter amplifier stage which determines the noise figure of the overall 2-stage amplifier. The second stage Darlington feedback amplifier provides broadband gain and output drive capability. The impedance bandwidth and reflection characteristic of the Darlington feedback stage can be optimized by controlling the series and parallel feedback resistors without degrading the noise performance of the overall amplifier. The shunt feedback resistor Rf1 of the Darlington stage can be control and vary as for as required gain bandwidth performance and Rf1 also gives biasing arrangement for transistor Q1 of the first stage. The shunt feedback resistor, Rf2, is used to optimized the noise and provide impedance matching, reduce contain of reflection and noise and improve bandwidth of amplifier. This technique gives better linearity of amplifier over the large bandwidth of amplifier. 


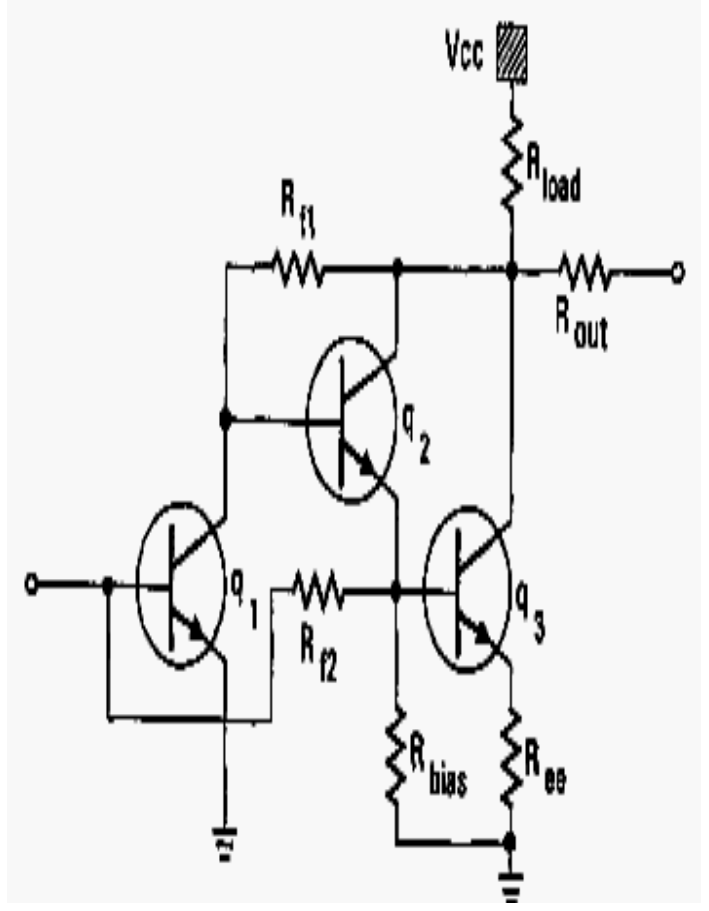

Fig. 1. Schematic of the direct-coupled HBT amplifier.

For further improvement in linearity, noise performance of amplifier next technique is discussed in next section.

\subsection{Resistive shunt feedback topology and Matching T-network sections}

Resistive shunt feedback topology and Matching T-network sections is versatile used in modern technology to easily give linearity and improved performance of amplifier. Broadband amplifier is more demanded and needed in distributed amplifier application. The problem of achieving a wideband impedance match to the transistor input and output impedance section is eliminate by incorporating the input and output coupling capacitances of a number of transistors into artificial transmission-line designing. But in recent times, for Ultra Wide Bandwidth system, the low power consumption inside the amplifier and linearity requirement generate a great challenge on low noise amplifier design. In this technique, shown in Fig. 2, the input stage of the LNA consists of a resistive shunt feedback technique with two T- Matching network sections gives impedance matching for $50 \Omega$ - antenna. This technique simultaneously will improve the noise performance and linearity of amplifier compared previous technique. The second stage is implemented in common source configuration to achieve the higher gain compared to common gate configuration. The output matching is generated by a single transistor, which means that the inductors eliminate the output capacitance of the second stage pHEMT and consists artificial transmission line terminated of $50 \Omega$ - to drive an external 50- load.

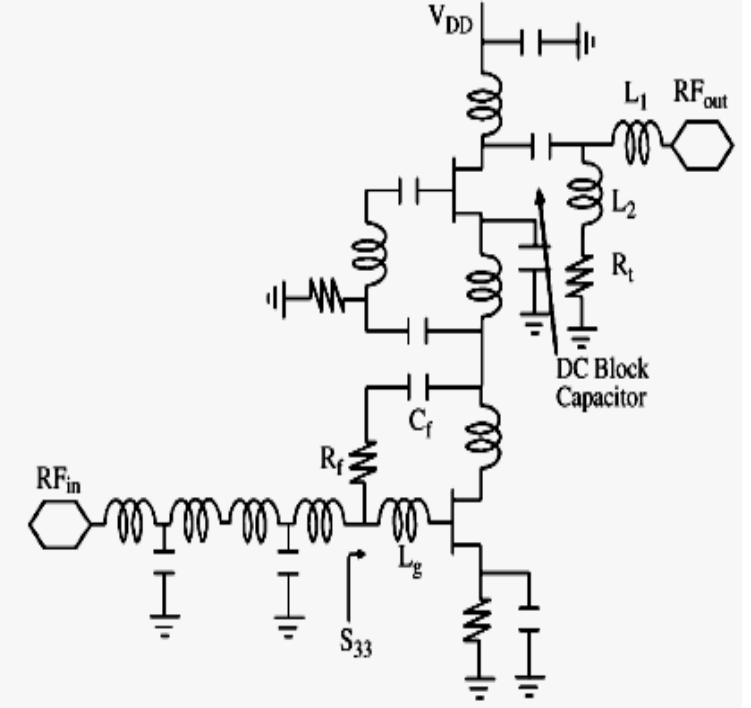

Fig. 2. Simplified schematic of the wideband LNA.

In order to reduce the power consumption and improve the noise performance; the number of the stages and element of the amplifier and the bias current are determined carefully. To make sure the transistor operates in the active region, the drain bias voltage of the transistor is at least 1.0 V. For further improvement in linearity, noise performance of amplifier next technique is discussed in next section.

\subsection{Forward Combining Technique}

The idea of forward combining technique is illustrated in Fig. 3 . The RF signals at the drain node and source node of the transistor are in anti-phase because they are derived from common-source and common-drain amplifications, respectively. The signal at drain node is shifted to have 90 phase advance and the signal at source node is shifted to have 90 phase lag. These two phase-shifted signals, which are in phase, are combined together before going into the next stage circuit .Since the two signals are in phase, the overall amplifier gain is boosted. The 90 phase shifts can simply be realized by an inductor and a capacitor. The noise figure of the amplifier can also be reduced through the gain enhancement because the noise resistance $\mathrm{Rn}$ is inversely proportional to the square of the trans-conductance

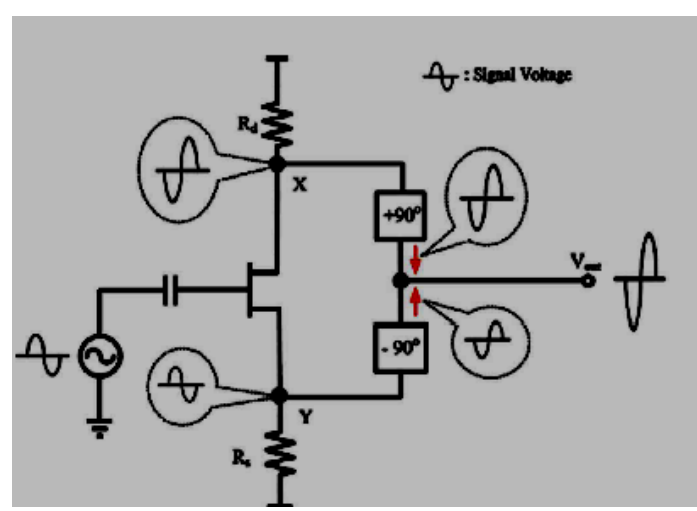

Fig. 3 Illustration of forward combining technique.

$$
R_{\mathrm{n}}=\frac{\gamma g_{a 0}}{g_{m}^{2}}
$$


For further improvement in linearity, noise performance of amplifier next technique is discussed in next section.

\subsection{Gate-Inductive Gain-Peaking Technique}

In this section gain boosting technique is discusses, utilize the gate-inductive gain-peaking scheme for the enhancement of maximum gain of amplifier. Inductor connects in series and shunt of proposed amplifier. This inductor offer very low resistance for designing and improve flow of current in output section of amplifier and improve linearity and performance of amplifier, in this technique number of component required is very less to achieved linearity, reduction in noise and reduction in power consumption. For further improvement in linearity, noise performance of amplifier next technique is discussed in next section.

\subsection{Switched multi-tap transformer and inductively degenerated}

In this technique implementing a transformer-based multimode Low Noise Amplifier that can be configured to operate in single-band, concurrent dual-band, or wideband frequency of application. In the conventional narrowband LNA, shown in Fig. 4, a series gate inductor, is used to achieved narrowband matching and high value of current,

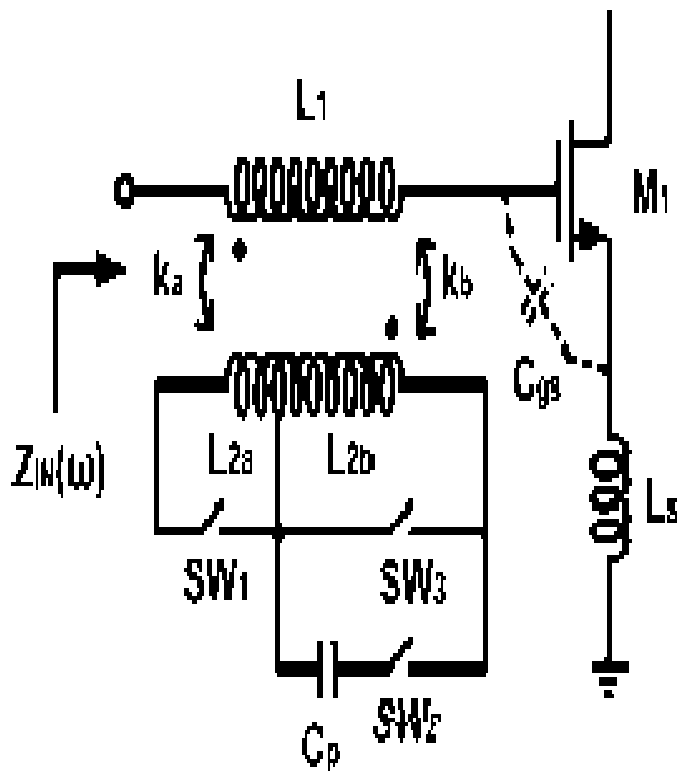

Fig 4 Multimode LNA with a reconfigurable multi-tap transformer as the gate inductor.

The input impedance of design is define as

$$
Z_{\mathrm{IN}}(\omega)=\frac{g_{m} L_{s}}{C_{\mathrm{gs}}}+j\left\{\omega L_{1}+\omega L_{s}-\frac{1}{\omega C_{\mathrm{gB}}}\right\}
$$

Where is the gm trans-conductance of transistor? From (1), the operating frequency is given as

$$
f=\frac{1}{2 \pi \sqrt{\left(L_{1}+L_{s}\right) C_{\mathrm{gs}}}} .
$$

By controlling the value of capacitance and inductance of amplifier the operating frequency of the LNA could be controlled easily making it suitable for use in applications for single band, multiband and dual band. For further improvement in linearity, noise performance of amplifier next technique is discussed in next section.

\section{7 $\mathrm{SiGe} \mathrm{BiCMOS} \mathrm{technology}$}

In this technique $\mathrm{SiGe}$ BiCMOS configuration is used to improve linearity and gain, and reduce the contain of noise and power consumption, the schematic of the two-stage LNA is shown in Fig. 5. The first stage is cascode is chosen for its higher power gain and improved input-to-output isolation and improve impedance matching for input and output section. By using such configuration, the noise performance and maximum power gain at high frequency are improved when compared with the higher single-base device (CBE) and larger parasitic capacitance triple base finger device (CBEBEBC). Common emitter (CE) with double-base finger device is used in the second stage to improved linearity and broader output matching bandwidth

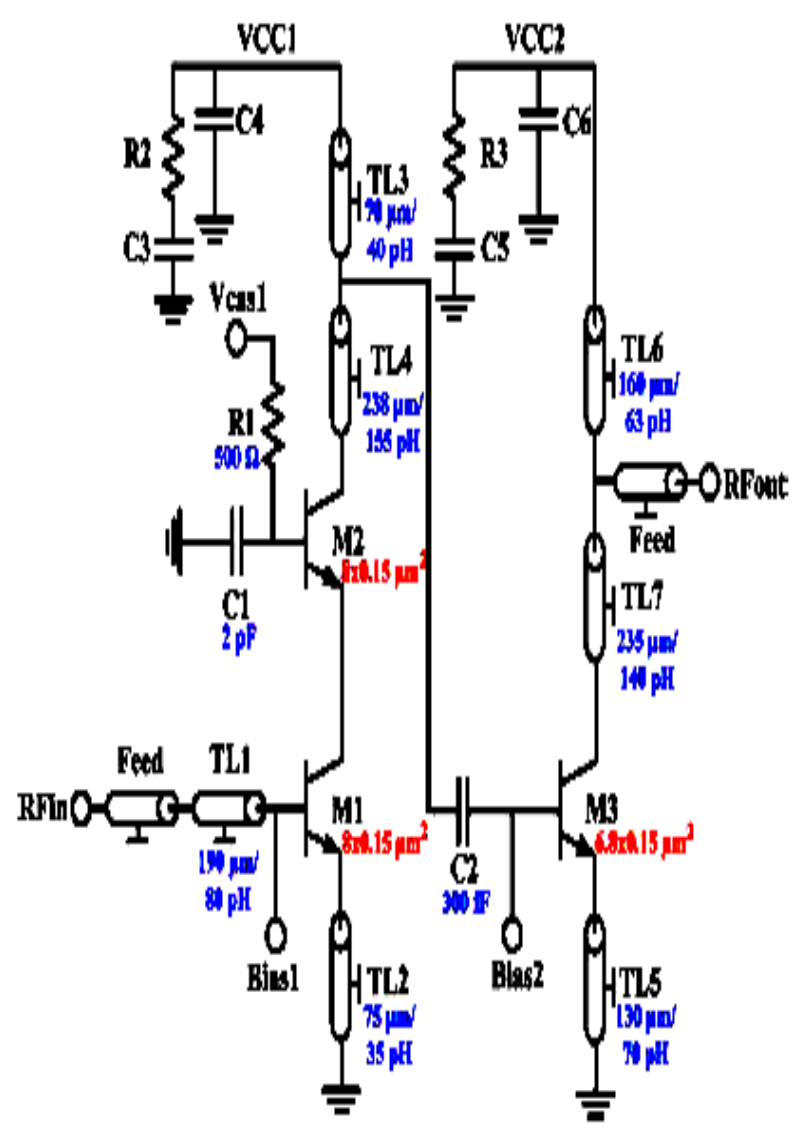

Fig. 5. Schematic of the two-stage low-noise amplifier 
Table 1 Review of Techniques

\begin{tabular}{|c|c|c|c|c|c|c|}
\hline Technique Used & $\begin{array}{c}\text { Power } \\
\text { Consump } \\
\text { tion }\end{array}$ & $\begin{array}{c}\text { S- } \\
\text { Parameter }\end{array}$ & $\begin{array}{c}\text { Broad } \\
\text { impedance } \\
\text { bandwidth }\end{array}$ & $\begin{array}{l}\text { Reduction in } \\
\text { Noise Figure }\end{array}$ & $\begin{array}{c}\text { Improve in } \\
\text { Linearity }\end{array}$ & Gain Boosting \\
\hline $\begin{array}{c}\text { Wide Range Derivative } \\
\text { Superposition } \\
\text { Technique }\end{array}$ & Moderate & Good & Good & Good & Good & Moderate \\
\hline $\begin{array}{c}\text { Direct-Coupled } \\
\text { Amplifier Topology }\end{array}$ & Moderate & Good & Moderate & Good & Good & Moderate \\
\hline $\begin{array}{c}\text { Resistive shunt } \\
\text { feedback topology and } \\
\text { Matching T-network } \\
\text { sections }\end{array}$ & less & Good & Good & Good & Good & Moderate \\
\hline $\begin{array}{c}\text { Forward Combining } \\
\text { Technique }\end{array}$ & Moderate & Good & Moderate & Good & Good & Good \\
\hline $\begin{array}{l}\text { Gate-Inductive Gain- } \\
\text { Peaking Technique }\end{array}$ & less & Good & Moderate & Good & Good & Good \\
\hline $\begin{array}{l}\text { Si-Ge Bi-CMOS } \\
\text { technology }\end{array}$ & less & Good & Moderate & Good & Good & Good \\
\hline $\begin{array}{c}\text { switched multi-tap } \\
\text { transformer and } \\
\text { inductively degenerated }\end{array}$ & Moderate & Good & Moderate & Good & Good & Good \\
\hline & & & & & & \\
\hline
\end{tabular}

\section{CONCLUSION}

Low noise amplifier versatile demanded and needed for recent technology like GSM, Wi-Max, WLAN, Satellite Communication, Mobile communication etc. in the review of Low Noise Amplifier (LNA), For improvement of the performance and linearity of low noise amplifier many number of techniques used in past decades are discuss like Wide Range Derivative Superposition Technique, DirectCoupled Amplifier Topology [2], Resistive shunt feedback topology and Matching T-network sections, Forward Combining Technique, Gate-Inductive Gain-Peaking Technique, Si-Ge Bi-CMOS technology, switched multi-tap transformer and inductively degenerated this all techniques discuss in details and concluded that product is versatile used in modern application. Discuss techniques to improve noise figure, reduce power consumption, gives good impedance matching in input and output section of amplifier, discuss about the gain boosting technique, inductive peaking is a best technique to improve the gain of amplifier, all techniques used to improve of LNA, improve gain, noise rejection capability and impedance bandwidth over single band, dual band, multi-band and broad band, but recent technology demand and needed low noise amplifier with versatile future so that more discussion and research required in field of low noise amplifier. Compare and discuss recent techniques in table-1, reviewed LNA from origin and discuss development and find Different technologies are used for implementing the different designs in the different aspects

\section{REFERENCES}

[1] Gao, W.,et.al A Highly Linear Low Noise Amplifier With Wide Range Derivative Superposition Method" Microwave and Wireless Components Letters, IEEE Year: 2015, Volume: 25, Issue: 12,Pages: 817 - 819,
[2] K. W. Kobayashi, Member, "A DC-10 GHz High GainLow Noise GaAs HBT Direct-Coupled Amplifier” IEEE Microwave And Guided Wave Letters, Vol. 5, No. 9, September 1995

[3] Yueh-Hua Yu, Student Member, IEEE, Wei-Hong Hsu, and Yi-Jan Emery Chen, Senior Member, IEEE" A KaBand Low Noise Amplifier Using Forward Combining Technique" IEEE Microwave And Wireless Components Letters, Vol. 20, No. 12, December 2010

[4] Chao Fang, Student Member, IEEE, “A 3.1-10.6 GHz Ultra-Wideband Low Noise Amplifier With 13-dB Gain, 3.4-dB Noise Figure , and Consumes Only $12.9 \mathrm{~mW}$ of DC Power" IEEE Microwave And Wireless Components Letters, Vol. 17, No. 4, April 2007295

[5] Chun-Chieh Huang, "Low-Power, High-Gain V-Band CMOS Low Noise Amplifier for Microwave Radiometer Applications.

[6] Xiaohua Yu, Student Member, 'Analysis and Design of a Reconfigurable Multimode Low-Noise Amplifier Utilizing a Multitap Transformer IEEE Transactions On Microwave Theory And Techniques, Vol. 61, No. 3, March 2013

[7] Austin Ying-Kuang Chen, "A Low-Power Linear SiGe BiCMOS Low-Noise Amplifier for Millimeter-Wave Active Imaging' IEEE Microwave And Wireless Components Letters, Vol. 20, No. 2, February 2010103

[8] J. Jin and S. Hsu, "A 0.18-nm CMOS balanced amplifier for 24-GHz applications," IEEE J. Solid-State Circuits, vol. 43, no. 2, pp. 440-445,Feb. 2008.

[9] S. Shin, M. Tsai, R. Liu, K. Lin, and H. Wang, "A 24 $\mathrm{GHz}$ 3.9-dB NF low-noise amplifier using $0.18 \mathrm{~nm}$ 
CMOS technology," IEEE Microw.Wireless Compon. Lett., vol. 15, no. 7, pp. 448-450, Jul. 2005.

[10] M. A. T. Sanduleanu, "31-34 GHz low noise amplifier with on-chip microstrip lines and inter-stage matching in 90-nm baseline CMOS," in Proc. IEEE RFIC Symp., 2006, pp.143-145.

[11] E. Adabi, B. Heydari, , "30 GHz CMOS low noise amplifier," in Proc. IEEE RFIC Symp., 2007, pp.625628.
[12] A. Sayag et al., "A $25 \mathrm{GHz} 3.3 \mathrm{~dB}$ NF low noise amplifier based upon slow wave transmission lines and the $0.18 \mathrm{~nm}$ CMOS technology," in Proc. IEEE RFIC Symp., 2008, pp. 373-376.

[13] Y. Wei, S. Hsu, and J. Jin, "A low-power low-noise amplifier for K-band applications," IEEE Microw. Wireless Compon. Lett., vol. 19,no. 2, pp. 116-118, Feb. 2009. 